\title{
STICK-SLIP TRANSITION CAPTURING BY USING AN ADAPTIVE FINITE ELEMENT METHOD
}

\author{
Nicolas Roquet ${ }^{1}$ and Pierre Saramito ${ }^{2}$
}

\begin{abstract}
The numerical modeling of the fully developed Poiseuille flow of a Newtonian fluid in a square section with slip yield boundary condition at the wall is presented. The stick regions in outer corners and the slip region in the center of the pipe faces are exhibited. Numerical computations cover the complete range of the dimensionless number describing the slip yield effect, from a full slip to a full stick flow regime. The resolution of variational inequalities describing the flow is based on the augmented Lagrangian method and a finite element method. The localization of the stick-slip transition points is approximated by an anisotropic auto-adaptive mesh procedure. The singular behavior of the solution at the neighborhood of the stick-slip transition point is investigated.
\end{abstract}

Mathematics Subject Classification. 65N22, 65N30, 65N50, 65Z05, 90C46.

Received: July 19, 2002.

\section{INTRODUCTION}

One of the difficult problems in numerical fluid computation is to describe slip at the boundary, especially when the slip is submitted to a yield value. In that case, the boundary condition is not linear. This situation occurs in many cases of practical interest: geophysical, food or petroleum fluid flows. In some cases, the material is not a Newtonian fluid: it would be viscoelastic or viscoelastic. Furthermore, the determination of the regions where the material slips or sticks are of practical interest, e.g. for earth cracks or for extrusion processes.

The fully developed flow in a tube with a square cross-section (see Fig. 1) contains the main features of such flows. In stick regions, located near the outward corners of the section, the shear stresses are low and the velocity vanishes at the boundary (see Fig. 2). Conversely, in slip regions, located at the center of the pipe faces, the shear stresses are higher and the fluid slips. Tube transition points between these two regions are called the stick-slip transition points.

To our knowledge, there are no numerical computations related to this subject. Our numerical strategy combines the augmented Lagrangian method that takes rigorously into account the slip yield stress constitutive equation, and the auto-adaptive mesh procedure for the capture of the stick-slip transition points.

Section 2 presents the slip boundary problem of the flow along a prismatic tube. The numerical method is presented in the third section. This is an adaptation to the slip yield condition of a numerical strategy presented in $[12-14]$ in the context of a Bingham fluid. The analogy between the slip yield condition and the Bingham

Keywords and phrases. Slip boundary condition, stick-slip problem, variational inequalities, adaptive mesh, computational fluid mechanics.

1 LMC-IMAG, BP 53, 38041 Grenoble Cedex 9, France.

2 LMC-IMAG, BP 53, 38041 Grenoble Cedex 9, France. e-mail: Pierre.Saramito@imag.fr 


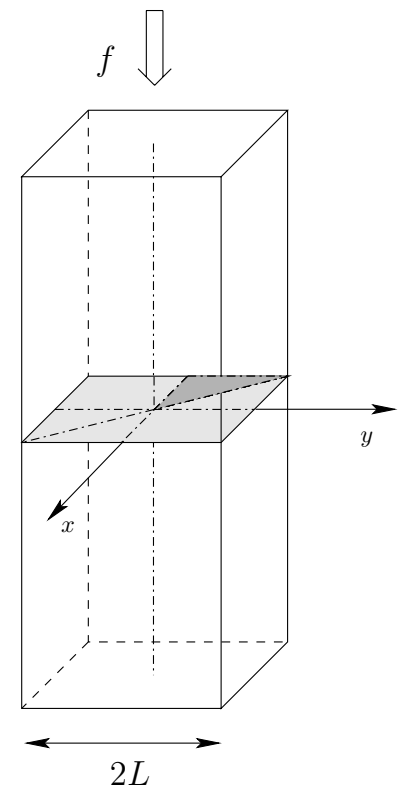

Figure 1. Square tube cross-section: tridimensional view.

yield stress constitutive equation allows us to extend the algorithms to the present case. Section 4 presents results on a square cross-section. Three flow regimes are exhibited and the evolution of the stick-slip transition point is carefully studied. The local behavior of the slip velocity at the neighborhood of the stick-slip transition point is analyzed.

\section{Problem statement}

\subsection{Constitutive equation and conservation laws}

Let $\sigma_{\text {tot }}$ denotes the total Cauchy stress tensor:

$$
\sigma_{\mathrm{tot}}=-p \cdot I+\sigma,
$$

where $\sigma$ denote its deviatoric part, and $p$ the pressure. The slip boundary condition reads:

$$
\mathbf{u}_{\mathbf{t}}=\left\{\begin{array}{cl}
-\left(1-\frac{s_{0}}{\left|\sigma_{\nu \mathbf{t}}\right|}\right) \frac{\sigma_{\nu \mathbf{t}}}{c_{f}}, & \text { when }\left|\sigma_{\nu \mathbf{t}}\right|>s_{0}, \\
0, & \text { otherwise }
\end{array}\right.
$$

where $\mathbf{u}$ is the velocity field, $s_{0} \geq 0$ the slip yield stress and $c_{f}>0$ the friction dissipation coefficient. The notations $\mathbf{u}_{\mathbf{t}}$ and $\sigma_{\boldsymbol{\nu} \mathbf{t}}$ are defined by

$$
\begin{aligned}
\mathbf{u}_{\mathbf{t}} & =\mathbf{u}-(\mathbf{u} . \boldsymbol{\nu}) \boldsymbol{\nu}, \\
\sigma_{\boldsymbol{\nu} \mathrm{t}} & =\sigma . \boldsymbol{\nu}-\left(\sigma_{\boldsymbol{\nu} \nu}\right) \boldsymbol{\nu},
\end{aligned}
$$

where $\sigma_{\boldsymbol{\nu} \boldsymbol{\nu}}=(\sigma \cdot \boldsymbol{\nu}) \cdot \boldsymbol{\nu}$ and $\boldsymbol{\nu}$ is the unit outward normal vector. For any vector field $\mathbf{v}$, the notation |.| represents the vector norm $|\mathbf{v}|=(\mathbf{v} . \mathbf{v})^{1 / 2}$. Notice that the vector field $\sigma_{\nu \mathbf{t}}$ is tangent to the boundary and that $\sigma_{\nu \nu}$ is a scalar field defined on the boundary. The slip relation can be also written as:

$$
\begin{array}{ll}
\sigma_{\boldsymbol{\nu} \mathbf{t}}=-c_{f} \mathbf{u}_{\mathbf{t}}-s_{0} \frac{\mathbf{u}_{\mathbf{t}}}{\left|\mathbf{u}_{\mathbf{t}}\right|}, & \text { if }\left|\mathbf{u}_{\mathbf{t}}\right| \neq 0, \\
\left|\sigma_{\boldsymbol{L} \mathbf{t}}\right| \leq s_{0}, & \text { if }\left|\mathbf{u}_{\mathbf{t}}\right|=0 .
\end{array}
$$




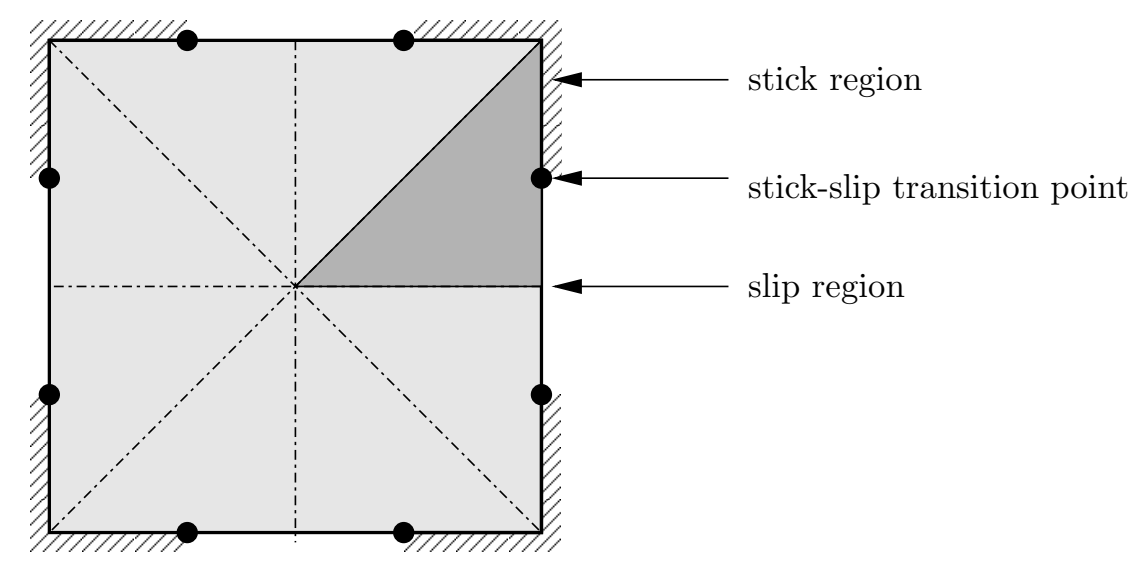

Figure 2. Schematic view of the cross-section.

The boundary condition is completed by a condition expressing that the fluid does not cross the boundary:

$$
\mathbf{u} . \nu=0 .
$$

Remark that for $s_{0}=0$, one obtains the classical linear slip boundary condition: the fluid slips for any nonvanishing shear stress $\sigma_{\nu \mathrm{t}}$. For $s_{0}>0$, boundary parts where the fluid sticks can be observed. As $s_{0}$ becomes larger, these stick regions develop. This simple law can be extended, as mentioned by Fortin et al. [5] or Ionescu and Vernescu [10]. In the context of solid mechanics and contact problems, Coulomb type friction has been studied by many authors. Refer e.g. to Haslinger et al. ([8], p. 377) for the numerical analysis and to Kikuchi and Oden [11] for the finite element approximation. In this case, the slip yield stress $s_{0}$ is no more a constant, and should be replaced by a quantity $s$ that depends upon the pressure at the boundary: $s=c_{0}\left|\sigma_{\nu \nu}\right|$. Nevertheless, previous works do not study the stick-slip transition. In this paper, since our purpose is to study a new numerical algorithms for the stick-slip transition capturing, we suppose that the slip yield stress is a constant.

The conservation of momentum is:

$$
\rho\left(\frac{\partial \mathbf{u}}{\partial t}+\mathbf{u} \cdot \nabla \mathbf{u}\right)-\operatorname{div} \sigma+\nabla p=0
$$

where $\rho$ is the constant density. Since the fluid is supposed to be incompressible, the mass conservation leads to:

$$
\operatorname{div} \mathbf{u}=0
$$

In this paper, the fluid is supposed to be Newtonian:

$$
\sigma=2 \eta D(\mathbf{u})
$$

here $D(\mathbf{u})=\left(\nabla \mathbf{u}+\nabla \mathbf{u}^{T}\right) / 2$ and $\eta>0$ is the constant viscosity.

We consider the fully developed flow in a prismatic tube (see Fig. 1). Let $(O z)$ be the axis of the tube and $(O x y)$ the plane of the bounded section $\Omega \subset \mathbb{R}^{2}$. The pressure gradient is written as $\nabla p=(0,0,-f)$ in $\Omega$, where $f>0$ is the constant applied force density. 


\subsection{The pipe flow problem}

The velocity is written as $\mathbf{u}=(0,0, u)$, where the third component $u$ along the $(O z)$ axis depends only upon $x$ and $y$, and is independent of $t$ and $z$. The problem can be written as a two-dimensional one:

$(P)$ : find $u$ defined in $\Omega$ such that

$$
\begin{aligned}
-\eta \Delta u & =f \text { in } \Omega, \\
u & =\left\{\begin{array}{cl}
-\left(1-\frac{s_{0}}{\eta \frac{\partial u}{\partial n} \mid}\right) \frac{\eta}{c_{f}} \frac{\partial u}{\partial n}, & \text { when }\left|\eta \frac{\partial u}{\partial n}\right|>s_{0} \\
0, & \text { otherwise }
\end{array}\right\} \text { on } \partial \Omega,
\end{aligned}
$$

where $\mathbf{n}$ is the unit outward normal vector on the boundary $\partial \Omega$ of the cross section $\Omega$.

Let $L$ be a characteristic length of the cross-section $\Omega$, e.g. the half-length of an edge of a square section. A characteristic velocity is given by $U=L^{2} f / \eta$. The slip yield dimensionless number $S$ is defined as the ratio of the slip yield stress $s_{0}$ to a characteristic stress $\Sigma=\eta U / L=L f$ :

$$
S=\frac{s_{0}}{L f}
$$

The friction dimensionless number $C_{f}$ is defined by

$$
C_{f}=\frac{c_{f} U}{\Sigma}=\frac{c_{f} L}{\eta} .
$$

The two dimensionless numbers $S$ and $C_{f}$ characterize the problem. The $C_{f}$ coefficient is chosen equal to the unity for all numerical experiments, and the $S$ parameter alone varies in this paper.

\section{Numerical Method}

The augmented Lagrangian method, applied to problem (1)-(2), is briefly introduced in this paragraph. Then, the delicate problem of the choice of a finite element approximation is carefully treated.

\subsection{Augmented Lagrangian algorithm}

Let $H^{1}(\Omega)$ denote the classical functional Sobolev space [1] and $J$ the convex functional defined for all $v \in H^{1}(\Omega)$ by

$$
J(v)=\frac{\eta}{2} \int_{\Omega}|\nabla v|^{2} \mathrm{~d} x+c_{f} \int_{\partial \Omega}|\gamma v|^{2} \mathrm{~d} s+s_{0} \int_{\partial \Omega}|\gamma v| \mathrm{d} s-\int_{\Omega} f v \mathrm{~d} x
$$

where $\mathrm{d} s$ is a measure on $\partial \Omega$ and $\gamma$ is the trace operator from $H^{1}(\Omega)$ to $H^{1 / 2}(\partial \Omega)$, i.e. $\gamma v$ is the restriction $v_{\mid \partial \Omega}$ of $v$ on $\partial \Omega$.

Using variational inequality methods (see e.g. Glowinski et al. [7]) we show that the solution $u$ of problem $(P)$ is characterized as the minimum of $J$ on $H^{1}(\Omega)$ :

$$
\min _{v \in H^{1}(\Omega)} J(v)
$$

Let us introduce the linear constraint

$$
\xi=\gamma u \in H^{1 / 2}(\partial \Omega)
$$


This constraint is handled by using a Lagrangian multiplier $\lambda \in L^{2}(\partial \Omega)$ that coincides with the shear stress $\boldsymbol{\sigma} . \mathbf{n}$ at the boundary. The Lagrangian $\mathcal{L}$ is defined for all $(u, \xi, \lambda) \in H^{1}(\Omega) \times\left(L^{2}(\partial \Omega)\right)^{2}$ by

$$
\begin{aligned}
\mathcal{L}(u, \xi ; \lambda)= & \frac{\eta}{2} \int_{\Omega}|\nabla u|^{2} \mathrm{~d} x-\int_{\Omega} f u \mathrm{~d} x \\
& +\frac{c_{f}}{2} \int_{\partial \Omega}|\xi|^{2} \mathrm{~d} s+s_{0} \int_{\partial \Omega}|\xi| \mathrm{d} s+\int_{\partial \Omega} \lambda(\xi-\gamma u) \mathrm{d} s
\end{aligned}
$$

For all $a>0$, the augmented Lagrangian

$$
\mathcal{L}_{a}(u, \xi ; \lambda)=\mathcal{L}(u, \xi ; \lambda)+\frac{a}{2} \int_{\partial \Omega}(\xi-\gamma u)^{2} \mathrm{~d} s
$$

is quadratic and positive-definite with respect to $u$. This implies that, with $\lambda$ and $\xi$ fixed, $\mathcal{L}_{a}$ can be minimized with respect to $u$ on $H^{1}(\Omega)$, whereas this operation is in practice impossible for $a=0$. This transformation proves to be helpful since we can solve the saddle-point problem of $\mathcal{L}_{a}$, that coincides with that of $\mathcal{L}$, by an appropriate algorithm proposed in [6]:

\section{Algorithm (Uzawa)}

initialization: $n=0$

Let $\lambda^{0}$ and $\xi^{0}$ be arbitrarily chosen in $L^{2}(\partial \Omega)$.

loop: $n \geq 0$

- step 1: suppose $\lambda^{n}$ and $\xi^{n}$ are known and find $u^{n+1} \in H^{1}(\Omega)$ such that

$$
\begin{aligned}
-\eta \Delta u^{n+1} & =f \quad \text { in } \Omega, \\
\eta \frac{\partial u^{n+1}}{\partial n}+a u^{n+1} & =\lambda^{n}+a \xi^{n} \text { on } \partial \Omega .
\end{aligned}
$$

- step 2: compute explicitly on $\partial \Omega$ :

$$
\xi^{n+1}:=\left\{\begin{array}{cl}
\left(1-\frac{\sigma_{0}}{\left|\lambda^{n}+a \gamma u^{n+1}\right|}\right) \frac{\lambda^{n}+a \gamma u^{n+1}}{c_{f}+a}, & \text { if }\left|\lambda^{n}+a \gamma u^{n+1}\right|>\sigma_{0} \\
0, & \text { otherwise. }
\end{array}\right.
$$

- step 3: compute explicitly on $\partial \Omega$ :

$$
\lambda^{n+1}:=\lambda^{n}+a\left(\xi^{n+1}-\gamma u^{n+1}\right)
$$

\section{end loop}

The advantage of this algorithm is that it transforms the global non-differentiable problem (5) into a family of completely standard problems (7)-(8) and a local explicit computation (9), coordinated via the Lagrange multiplier in (10). The sequence $\left(u^{n}, \xi^{n}, \lambda^{n}\right)$ converges for all $a>0$ to $(u, \xi, \lambda)$ where $u \in H^{1}(\Omega)$ is the solution to $(5)$ and $\xi=u_{\mid \partial \Omega}$ and $\lambda=\eta \frac{\partial u}{\partial n}$ on $\partial \Omega$. 


\subsection{Finite element approximation}

Let $A$ and $B$ be the two bilinear forms defined by:

$$
\begin{aligned}
& A(u, \xi ; v, \zeta)=\eta \int_{\Omega} \nabla u . \nabla v \mathrm{~d} x+\left(c_{f}+a\right) \int_{\partial \Omega} \gamma u \gamma v \mathrm{~d} s+a \int_{\partial \Omega}(\xi \zeta-\gamma u \zeta-\xi \gamma v) \mathrm{d} s \\
& B(v, \zeta ; \mu)=\int_{\partial \Omega} \mu(\zeta-\gamma v) \mathrm{d} s .
\end{aligned}
$$

The saddle point of $\mathcal{L}_{a}$ is characterized as the solution of a problem expressed by the following variational inequalities:

$(V I):$ find $(u, \xi ; \lambda) \in H^{1}(\Omega) \times\left(L^{2}(\partial \Omega)\right)^{2}$ such that:

$$
\begin{aligned}
s_{0} \int_{\partial \Omega}(|\zeta|-|\xi|) \mathrm{d} s+A(u, \xi ; v, \zeta-\xi)+B(v, \zeta-\xi ; \lambda) & \geq \int_{\Omega} f v \mathrm{~d} x, \\
B(u, \xi ; \mu) . & =0
\end{aligned}
$$

for all $(v, \zeta ; \mu) \in H^{1}(\Omega) \times\left(L^{2}(\partial \Omega)\right)^{2}$.

Let $V_{h} \subset H^{1}(\Omega), \Xi_{h} \subset L^{2}(\partial \Omega)$, and $\Lambda_{h} \subset L^{2}(\partial \Omega)$ be some finite dimensional spaces. The finite dimensional version of the variational inequalities is simply obtained by replacing functional spaces by their finite dimensional counterparts:

$(V I)_{h}:$ find $\left(u_{h}, \xi_{h} ; \lambda_{h}\right) \in V_{h} \times \Xi_{h} \times \Lambda_{h}$ such that:

$$
\begin{aligned}
\sigma_{0} \int_{\partial \Omega}\left(|\zeta|-\left|\xi_{h}\right|\right) \mathrm{d} s+A\left(u_{h}, \xi_{h} ; v, \zeta-\xi_{h}\right)+B\left(v, \zeta-\xi_{h} ; \lambda_{h}\right) & \geq \int_{\Omega} f v \mathrm{~d} x \\
B\left(u_{h}, \xi_{h} ; \mu\right) & =0
\end{aligned}
$$

for all $(v, \zeta ; \mu) \in V_{h} \times \Xi_{h} \times \Lambda_{h}$.

The choice $\Xi_{h}=\Lambda_{h}$ leads to $\xi_{h}=R_{h} \gamma u_{h}$ where $R_{h}$ denotes the projection from $L^{2}(\partial \Omega)$ on $\Lambda_{h}$, defined for all $\varphi \in L^{2}(\Omega)$ by:

$$
R_{h} \varphi \in \Lambda_{h} \text { and } \int_{\partial \Omega} R_{h} \varphi \zeta \mathrm{d} s=\int_{\partial \Omega} \varphi \zeta \mathrm{d} s, \quad \forall \zeta \in \Lambda_{h}
$$

For $s_{0}=0$, the problem reduces to a linear one that fits the theory of mixed finite elements (see e.g. [3]). See also [4] for the use of the properties of $R_{h}$ in the context of stabilized mixed finite element approximation. The case $s_{0}=0$ together with the choice $\Xi_{h}=\Lambda_{h}$ leads to $\lambda_{h}=-c_{f} R_{h} \gamma u_{h}$ and the problem reduces to the following linear elliptic one:

$(Q)_{h}$ find $u_{h} \in V_{h}$ such that

$$
\eta \int_{\Omega} \nabla u_{h} \cdot \nabla v \mathrm{~d} x+c_{f} \int_{\partial \Omega} R_{h} \gamma u_{h} R_{h} \gamma v \mathrm{~d} s+a \int_{\partial \Omega}\left(I-R_{h}\right) \gamma u_{h}\left(I-R_{h}\right) \gamma v \mathrm{~d} s=\int_{\Omega} f v \mathrm{~d} x, \quad \forall v \in V_{h} .
$$

When $R_{h} \neq I$ i.e. $\Lambda_{h} \neq \gamma V_{h}$, the discrete solution $u_{h}$ depends upon the numerical parameter $a>0$ of the augmented Lagrangian method. This property is not desirable. A necessary and sufficient condition for the solution $u_{h}$ to be independent of the parameter $a$ is therefore $\Lambda_{h}=\gamma V_{h}$.

Let $\mathcal{T}_{h}$ be a finite element mesh made up of triangles and let $\partial \mathcal{T}_{h}$ denote the corresponding mesh of the boundary $\partial \Omega$, consisting in segments. We introduce the space $V_{h}$ of continuous piecewise polynomials of order $k \geq 1$, relative to $\mathcal{T}_{h}$ :

$$
V_{h}=\left\{v \in H^{1}(\Omega) ; v_{\mid K} \in P_{k}, \forall K \in \mathcal{T}_{h}\right\}
$$

Thus, $\Lambda_{h}=\Xi_{h}=\gamma V_{h}$ is the set of continuous piecewise polynomial functions defined on the mesh boundary $\partial \mathcal{T}_{h}$

$$
\Xi_{h}=\Lambda_{h}=\left\{\mu \in L^{2}(\partial \Omega) \cap C^{0}(\partial \Omega) ; \mu_{\mid S} \in P_{k}, \forall S \in \partial \mathcal{T}_{h}\right\}
$$

Numerical experiments presented in this paper use piecewise linear polynomials, i.e. $k=1$. 


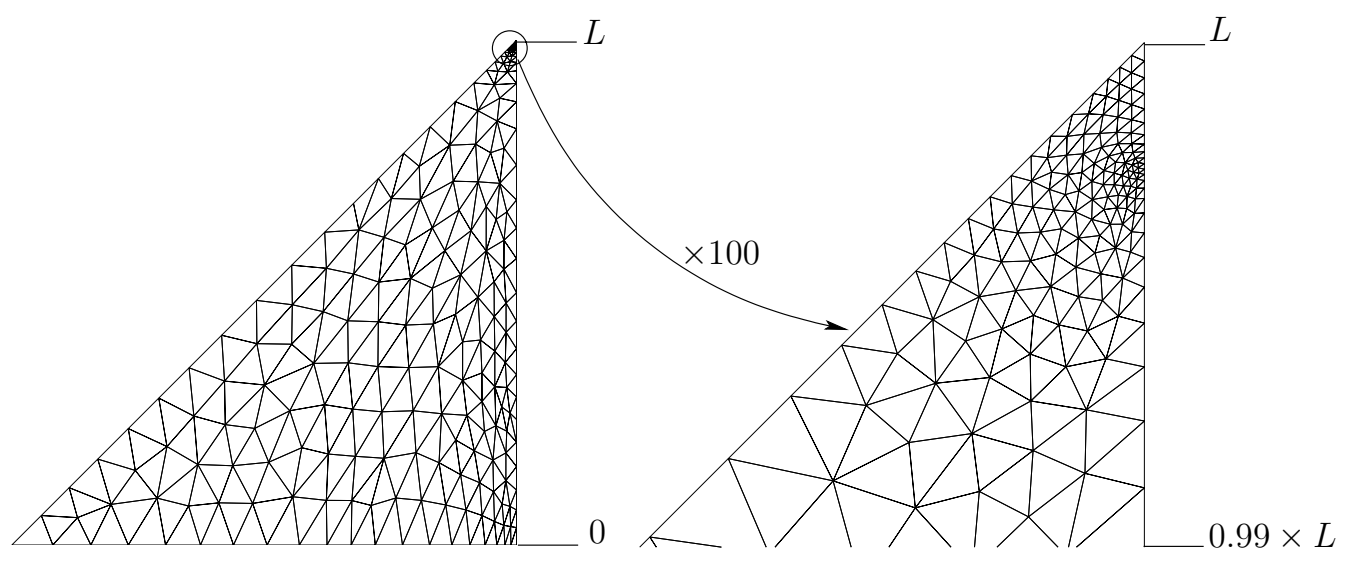

FigurE 3 . Zoom $\times 100$ at the neighborhood of the stick-slip transition point: after 15 mesh adaptation iterations $(S=0.385)$.

\subsection{Mesh adaptation}

The mesh adaptation procedure has already been described in [12,14] for a Bingham fluid flow problem, and thus, only the main steps are presented in this paragraph.

Following Vallet [17], a way to adapt the mesh to the computation of a governed field is to equi-distribute its error of interpolation, i.e. to make it constant over all triangles and in all directions. Solving a problem using a mesh adaptation is an iterative process, which involves three main steps:

1. Starting from an initial mesh $\mathcal{T}_{0}$, the problem is solved using the augmented Lagrangian algorithm, yielding a solution $u^{(0)}$. to the mesh $\mathcal{T}_{0}$.

2. Let $\varphi^{(0)}=\left|\nabla u^{(0)}\right|$ be the governing field. This field emphasizes regions where the solution has high derivatives, so that the mesh generator refines these regions.

3. Starting from the governing field $\varphi^{(0)}$ on the mesh $\mathcal{T}_{0}$, an anisotropic adaptive mesh generator (see Borouchaki et al. [2], Hecht [9]) generates a totally new mesh, denoted by $\mathcal{T}_{1}$.

Then, $\mathcal{T}_{1}$ is used to solve the problem, and so on, until the solution obtained reaches an accurate localization of the stick-slip transition point. This method is based on the fact that high second derivatives of the velocity develop at the neighborhood of the stick-slip transition point, and thus the mesh generator refines this neighborhood. The singular behavior of the second derivative of the velocity at the neighborhood of the transition point will be analyzed in detail in the next section. The software is based on a finite element library released by the authors $[15,16]$.

In order to reduce the computational cost in the square cross-section, we exploit the symmetries of the solutions with respect to the $O x, O y$ and the $x=y$ axis. Thus the domain of computation reduces to a triangle (see again Fig. 2). Figure 3 shows the mesh after 15 adaptation loops and for a slip yield number $S=0.385$, as defined in (3). The stick-slip transition point is close to the corner $x=y=L$, and the stick region is small. Observe that the mesh adaptation process is able to catch the stick-slip transition point.

\section{Numerical EXPERIMENTS ON THE SQUARE SECTION}

\subsection{Flow regimes}

Observe in Figure 4a the velocity profile along the boundary $u_{\mid \partial \Omega}(y)=u(L, y)$ for various values of $S$. When $S$ increases, the stick region develops. Each profile is a decreasing curve, with a vanishing tangent at the origin. Moreover, at each point of the boundary, the velocity is a decreasing function of $S$. Let us denote by $y_{t}(S) \in[0, L]$ the stick-slip transition point. The slip region is then defined by $\left[0, y_{t}(S)[\right.$ and the stick region by 


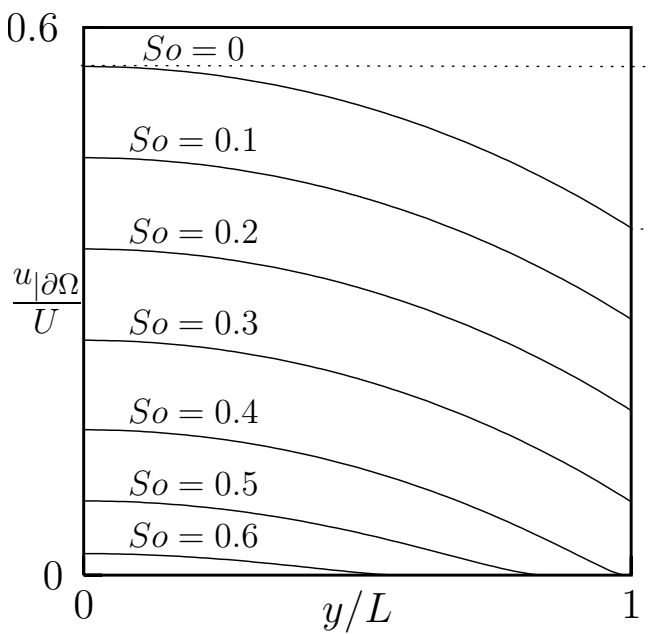

(a)

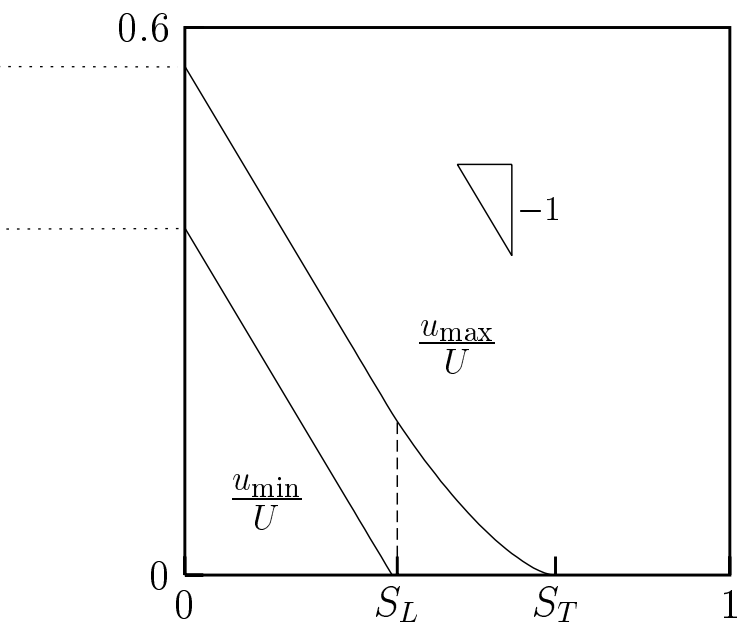

(b)

Figure 4. Slip velocity on the boundary: (a) for various $S$ values; (b) extremal slip velocities as a function of $S$.

]$\left.y_{t}(S), L\right]$. Remark that there exists $S$ values with empty stick region, for instance $S=0$. Conversely, for some other values of $S$, slip regions are empty. Since the velocity is decreasing with $S$, there are three flow regimes:

1. A full slip regime: the fluid slips all along the boundary. This regime is characterized by $S \leq S_{L}$, where $S_{L} \approx 0.382$ is the threshold value ( $S_{L}$ stands for slip).

2. A full stick regime: the fluid sticks all along the boundary. This regime is characterized by $S \geq S_{T}$, where $S_{T} \approx 0.674$ is the limit value (Soa stands for stick).

3. A mixed stick-slip regime: there exists a stick-slip transition point $\left.y_{t}(S) \in\right] 0, L[$. This regime is characterized by $S_{L}<S<S_{T}$.

A dichotomy algorithm has been used to obtain the approximate values of $S_{L}$ and $S_{T}$.

In the full slip regime, the solution is obtained by a simple translation from the solution $u_{0}$ associated to $S=0$ :

$$
u_{S}(x, y)=u_{0}(x, y)-S, \quad 0 \leq S \leq S_{L}
$$

Recall that the problem for $S=0$ is a linear Poisson problem with Robin boundary conditions:

$$
-\eta \Delta u_{0}=f \text { in } \Omega \text { and } \eta \frac{\partial u_{0}}{\partial n}+c_{f} u_{0}=0 \text { on } \partial \Omega
$$

In the full stick regime, the solution does not depend upon $S$ :

$$
u_{S}(x, y)=u_{\infty}(x, y), \quad S \geq S_{T}
$$

where $u_{\infty}$ is obtained by solving a linear Poisson problem with Dirichlet boundary conditions:

$$
-\eta \Delta u_{\infty}=f \text { in } \Omega \text { and } u_{\infty}=0 \text { on } \partial \Omega .
$$

Thus, the problem reduces to the study of the mixed stick-slip regime $S_{L}<S<S_{T}$.

Then, observe in Figure $4 \mathrm{~b}$ the evolution of the minimal and maximal velocity at the boundary as a function of $S$ :

$$
u_{\max }(S)=u_{S}(L, 0) \quad \text { and } \quad u_{\min }(S)=u_{S}(L, L)
$$




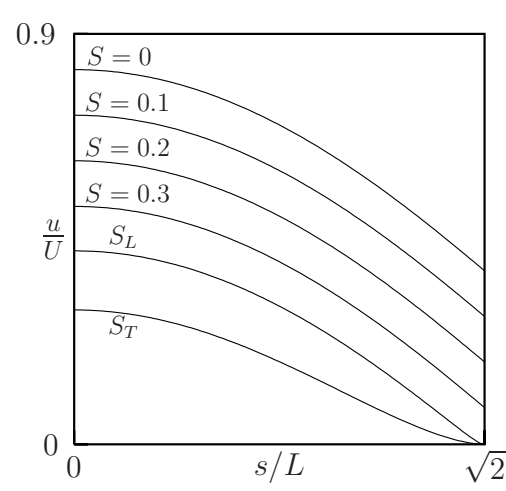

(a)

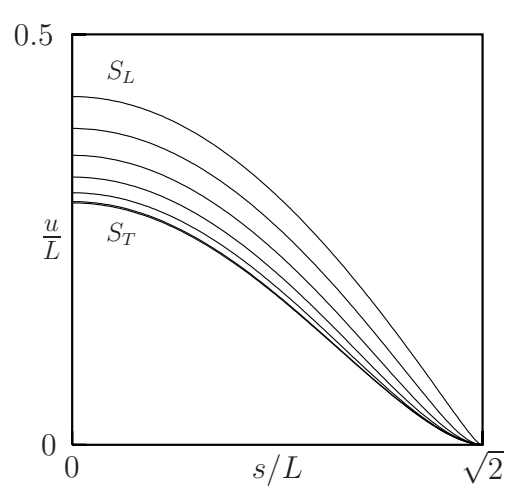

(c)

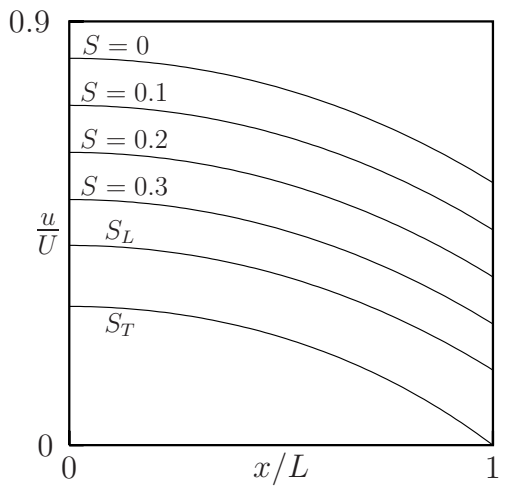

(b)

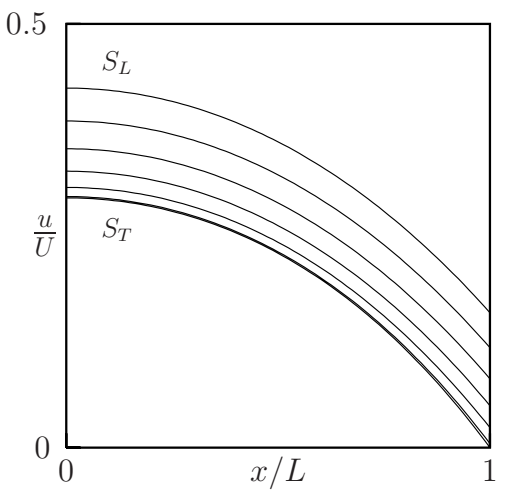

(d)

FIGURE 5. Velocity profile along the symmetry axes for various $S$ values: (a) along the diagonal symmetry axis for $0 \leq S \leq S_{T}$; (b) along the horizontal symmetry axis for $0 \leq S \leq S_{T}$; (c) along the diagonal symmetry axis for $S_{L} \leq S \leq S_{T}$; (d) along the horizontal symmetry axis for $S_{L} \leq S \leq S_{T}$.

In the mixed stick-slip regime, $u_{\max }$ is no more an affine function of $S$ and seems to vanish at $S=S_{T}$ with an horizontal slope.

Observe in Figures $5 \mathrm{a}$ and $5 \mathrm{~b}$ the velocity profiles along symmetry axes. Again, the plots show that $u_{S}=$ $u_{0}-S$ for $S \geq S_{L}$. When $S$ increases from $S_{L}$ to $S_{T}$ (Figs. $5 \mathrm{c}$ and $5 \mathrm{~d}$ ) the profiles decrease and tend to the profiles associated to $S=S_{T}$. For greater values of $S$, the velocity does not change any more.

\subsection{The neighborhood of the stick-slip point}

Figure 6 shows the first and the second tangential derivatives of the slip velocity along the boundary. Figure $6 \mathrm{a}$ suggests that the tangential derivative $\frac{\partial u_{\mid \partial \Omega}}{\partial t}$ shows a vertical slope, as we can check in Figure $6 \mathrm{~b}$, where the second derivative exhibits a peak at the stick-slip transition point.

Figure 7 plots the slip velocity along the boundary at the neighborhood of the stick-slip transition point by using a logarithmic scale. Observe the asymptotic behavior for $y \approx y_{t}(S)$, which seems to obey to a power law. Thus, let us look at the neighborhood of $y=y_{t}(S), y<y_{t}(S)$ for

$$
u_{S} \approx c(S) \times\left\{y_{t}(S)-y\right\}^{\alpha(S)}
$$




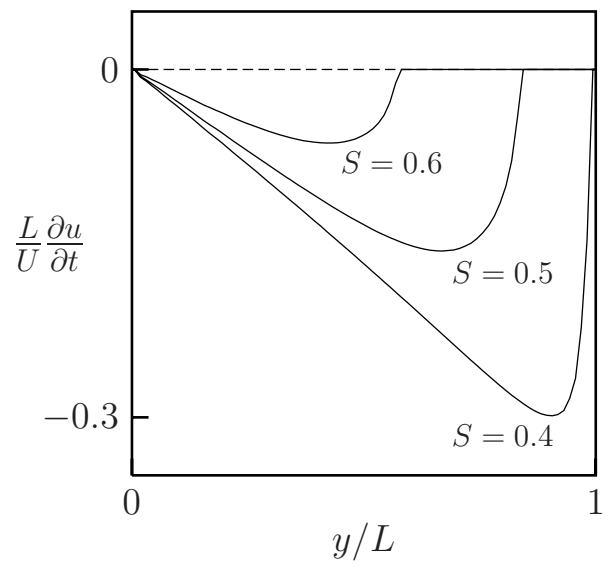

(a)

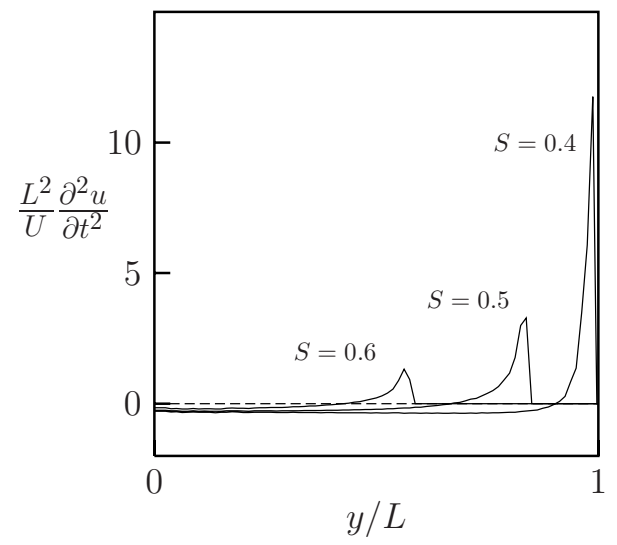

(b)

FiguRE 6. First (a) and second (b) tangential derivatives of the slip velocity along the boundary.

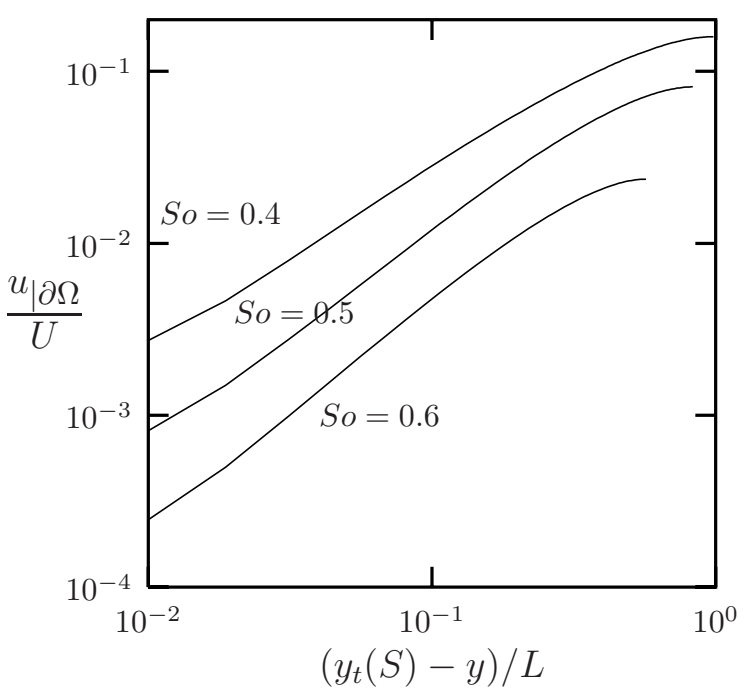

Figure 7 . Behavior of the slip velocity at the neighborhood of the stick-slip boundary point.

For each value of $S$, the values for $\alpha(S)$ and $c(S)$ are computed numerically by a non-linear least square fitting procedure. The coefficient $\alpha$ is provided in the Table 1 together with the stick-slip location point $y_{t}(S)$ for $S=0.4,0.5$ and 0.6. The second tangential derivative is singular at the neighborhood of the stick-slip transition point:

$$
\frac{\partial^{2} u_{S}}{\partial t^{2}} \approx k(S) \times\left\{y_{t}(S)-y\right\}^{\alpha(S)-2} .
$$

Since $\alpha(S)$ increases with $S$, the singularity is stronger for small values of $S$, i.e. at the neighborhood $S \approx S_{L}$, $S>S_{L}$, as we can observe in Figure 6b.

Figure 8 shows the stick-slip transition point as a function of $S$. The slope seems to be vertical in $S=S_{T}$ : when $S$ decreases and reaches the $S_{T}$ values, a slip occurs and the stick-slip transition point $y_{t}$ goes rapidly away from the neighborhood of $y_{t}=0$ : The associated stick region $\left[0, y_{t}\right]$ grows very fast. 
TABle 1. Coefficient $\alpha(S)$ at the stick-slip point transition $y_{t}(S)$.

\begin{tabular}{|l|l|l|}
\hline$S$ & $\alpha(S)$ & $y_{t}(S)$ \\
\hline \hline 0.4 & 1.08 & 0.98 \\
\hline 0.5 & 1.25 & 0.83 \\
\hline 0.6 & 1.33 & 0.57 \\
\hline
\end{tabular}

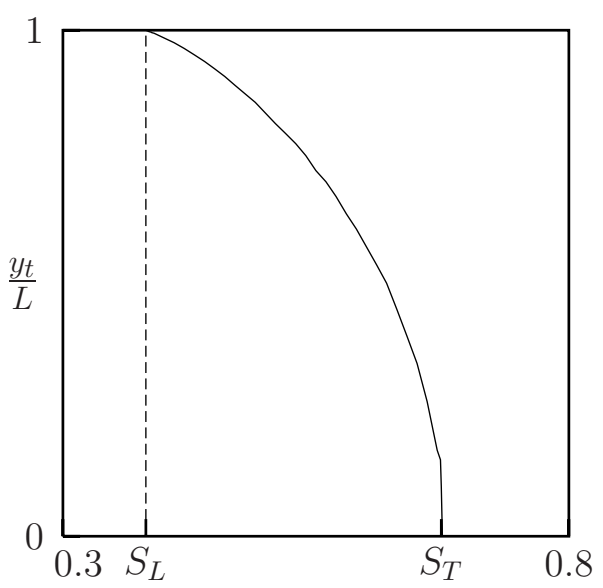

Figure 8. Coordinate of the stick-slip transition point $y_{t}(S)$ as a function of $S$.

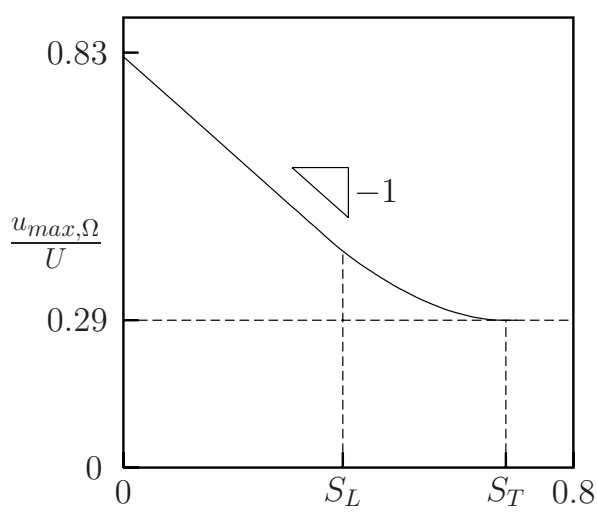

(a)

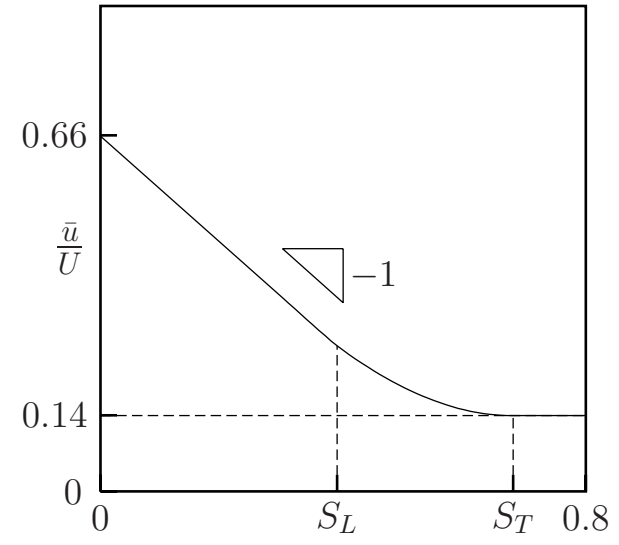

(b)

Figure 9. Maximal (a) and mean (b) velocities as a function of $S$.

\section{Global maximal And mean values}

Figure 9 plots the maximal and mean velocities as a function of $S$. These curves are affine in the full slip regime, gradually varied in the stick-slip regime, and becomes constant for the full stick regime $S=S_{T}$ with an horizontal slope. Despite the appearance of the stick-slip transition point in the mixed stick-slip flow regime, the maximal and mean values depend smoothly on $S$. 


\section{Conclusion}

A new numerical method for slip yield boundary conditions as been addressed in this paper. Such problems are characterized by the existence of a stick-slip transition point located on the boundary. The location of this point depends upon the parameters of the problem and is a priori unknown. An important result is the accurate capture of this transition point and the determination of three flow regimes.

The results of the present paper extend results [12-14] that have been developed in the context of Bingham fluids: the combination of an adaptive mesh strategy and the augmented Lagrangian method. Applied here to a square cross-section, the present numerical procedure can be applied to a tube section of any shape.

Slip yield boundary conditions is of practical interest and great importance in many geophysical and industrial applications involving complex materials. In the future, we will turn to non-Newtonian fluids problems involving slip yield boundary conditions.

\section{REFERENCES}

[1] R.A. Adams, Sobolev spaces. Academic Press (1975).

[2] H. Borouchaki, P.L. George, F. Hecht, P. Laug and E. Saltel, Delaunay mesh generation governed by metric specifications. Part I: Algorithms. Finite Elem. Anal. Des. 25 (1997) 61-83.

[3] F. Brezzi and M. Fortin, Mixed and Hybrid Finite Element Methods. Springer Verlag (1991).

[4] F. Brezzi, M. Fortin and R. Stenberg, Error analysis of mixed-interpolated elements for Reissner-Mindlin plates. Research Repport No. 780, Instituto di Analisi Numerica, Pavie (1991).

[5] A. Fortin, D. Côté and P.A. Tanguy, On the imposition of friction boundary conditions for the numerical simulation of Bingham fluid flows. Comput. Meth. Appl. Mech. Engrg. 88 (1991) 97-109.

[6] M. Fortin and R. Glowinski, Méthodes de lagrangien augmenté. Applications à la résolution numérique de problèmes aux limites. Méthodes Mathématiques de l'Informatique, Dunod (1982).

[7] R. Glowinski, J.L. Lions and R. Trémolières, Numerical analysis of variational inequalities. North Holland, Amsterdam (1981).

[8] J. Haslinger, I. Hlavàček and J. Nečas, Numerical methods for unilateral problems in solidmechanics. P.G. Ciarlet and J.L. Lions Eds., Handb. Numer. Anal. IV (1996).

[9] F. Hecht, Bidimensional anisotropic mesh generator. INRIA (1997). http://www-rocq.inra.fr/gamma/cdrom/www/bamg

[10] I.R. Ionescu and B. Vernescu, A numerical method for a viscoplastic problem. An application to the wire drawing. Int. J. Engrg. Sci. 26 (1988) 627-633.

[11] N. Kikuchi and J.T. Oden, Contact problems in elasticity: A study of variational inequalities and finite element methods. SIAM Stud. Appl. Math. (1988).

[12] N. Roquet and P. Saramito, An adaptive finite element method for Bingham fluid flows around a cylinder. Comput. Methods Appl. Mech. Engrg. 192 (2003) 3317-3341.

[13] N. Roquet, R. Michel and P. Saramito, Errors estimate for a viscoplastic fluid by using $P_{k}$ finite elements and adaptive meshes. C. R. Acad. Sci. Paris, Série I 331 (2000) 563-568.

[14] P. Saramito and N. Roquet, An adaptive finite element method for viscoplastic fluid flows in pipes. Comput. Methods Appl. Mech. Engrg. 190 (2001) 5391-5412.

[15] P. Saramito and N. Roquet, Rheolef home page. http://www-lmc.imag.fr/lmc-edp/Pierre.Saramito/rheolef/ (2002).

[16] P. Saramito and N. Roquet, Rheolef users manual. Technical report, LMC-IMAG (2002). http://www-lmc.imag.fr/lmc-edp/ Pierre.Saramito/rheolef/usrman.ps.gz

[17] M.G. Vallet, Génération de maillages anisotropes adaptés. Application à la capture de couches limites. Rapport de Recherche No. 1360, INRIA (1990). 